Article

\title{
Variations in Daily Maximum Areas and Longitudinal Widths of Solar Coronal Holes in 2017-2020
}

\author{
Alexandr Riehokainen ${ }^{1, *(D)}$, Victoria Smirnova ${ }^{2}$, Alexander Solov'ev ${ }^{3,4}$, Andrey Tlatov ${ }^{3}$, Ivan Zhivanovich $^{3}$, \\ Firas Al-Hamadani ${ }^{5}$ and Polina Strekalova ${ }^{3}$
}

\section{check for} updates

Citation: Riehokainen, A.; Smirnova, V.; Solov'ev, A.; Tlatov, A.; Zhivanovich, I.; Al-Hamadani, F.; Strekalova, P. Variations in Daily Maximum Areas and Longitudinal Widths of Solar Coronal Holes in 2017-2020. Universe 2022, 8, 158. https://doi.org/ $10.3390 /$ universe 8030158

Academic Editors: Juha Kallunki, Arnold Hanslmeier and Athanasios Papaioannou

Received: 3 December 2021 Accepted: 22 February 2022 Published: 2 March 2022

Publisher's Note: MDPI stays neutral with regard to jurisdictional claims in published maps and institutional affiliations.

Copyright: (C) 2022 by the authors. Licensee MDPI, Basel, Switzerland. This article is an open access article distributed under the terms and conditions of the Creative Commons Attribution (CC BY) license (https:// creativecommons.org/licenses/by/ $4.0 /)$.
1 Department of Physics and Astronomy, University of Turku, 20014 Turku, Finland

2 Crimean Astrophysical Observatory, Crimea, 298409 Nauchny, Russia; vvsvid.sm@gmail.com

3 Central (Pulkovo) Astronomical Observatory RAS, 196140 St-Petersburg, Russia; solov.a.a@mail.ru (A.S.); tlatov@mail.ru (A.T.); ivanzhiv@live.com (I.Z.); auriga-lynx@yandex.ru (P.S.)

4 The Faculty of Mathematics, Physics and Information Technologies, Kalmyk State University, 358000 Elista, Russia

5 Department of Physics, University of Basrah, Basrah 61004, Iraq; firas.balbool@uobasrah.edu.iq

* Correspondence: alerie@utu.fi

\begin{abstract}
We considered coronal holes as a manifestation of the large-scale magnetic field of the Sun. The main goal of this work was to study the variations in the largest areas and longitudinal widths of solar coronal holes observed daily in the polar and mid-latitude zones on a time scale of 984 days. Statistical methods of fast Fourier transform (FFT), wavelet transform, and empirical mode decomposition (EMD) were used to detect periodicity in the variations of the considered parameters. Long-term variations in the daily measured areas and longitudinal widths of the largest solar coronal holes with periods of 8-9, 13-15, and 26-29 days were detected in three zones of the Sun: polar (north and south) and equatorial. The obtained periods are most clearly visible at the equatorial zone. In the polar zones the period of 8-9 days has a weak amplitude. We interpreted variations with periods of 8-9, 13-15, and 26-29 days as a rotation of the six-, four-, and two-sector structure of the large-scale solar magnetic field.
\end{abstract}

Keywords: Sun; coronal holes; magnetic field; rotation

\section{Introduction}

Coronal holes (CHs) are structures with an open configuration of a magnetic field. Due to this configuration of the magnetic field, charged particles can escape from the corona, forming solar wind (for more details see $[1,2]$ ), which has an important effect on space weather [3]. The study of the $\mathrm{CH}$ structures, their origin, and various physical parameters is important for understanding the complex processes of cyclic solar activity.

Coronal holes are visible throughout the solar disk. They can be divided into polar and mid-latitude (or equatorial) coronal holes.

Polar CHs are one of the longest-lived structures of the Sun; in accordance with [4], equatorial coronal holes may live more than 18 months and polar coronal holes may live 2-3 years. They contribute to the global state of the solar corona and can be associated with the large-scale structure of the magnetic field. Even though $\mathrm{CHs}$ have been studied for a long time, not all of their parameters are clearly defined. Extensive coronal hole parameters over a whole solar cycle (including area) are given in [5] and plasma properties in [6]. Coronal hole parameters such as the magnetic field strength are also given in $[7,8]$; rotation speed [9], frequency of occurrence, evolution of areas during the solar cycle [4], height structure, and physical parameters such as temperature, density, and others have been intensively studied.

Numerous works have been devoted to the study of the identification of $\mathrm{CH}$ height structure [10], as well as the propagation of magnetohydrodynamic (MHD) waves [11,12] 
in them. There are some works devoted to the establishing of the physical parameters of a $\mathrm{CH}[13,14]$. Observations of $\mathrm{CHs}$ show that these are regions with low plasma density, and their temperature is close to the temperature of the surrounding corona [14].

In [15], the authors investigated the relationship between the $\mathrm{CH}$ properties and their variations in the solar cycle using cycles 20-23, comparing the change in $\mathrm{CH}$ areas with the change in the length of solar filaments and sunspot areas. The authors also pointed out the presence of an asymmetry in $\mathrm{CH}$ activity between the northern and southern polar zones. A few works are devoted to the study of $\mathrm{CH}$ rotation in accordance with the sunspot number [16]. Studies [17] found a relationship between long-lived isolated $\mathrm{CH}$ and solar flares observed in the soft $X$-ray range.

The formation of $\mathrm{CHs}$, which is related to the magnetic field configuration, is described in [18]. The relationship between variations in the areas of polar $\mathrm{CHs}$ with a large-scale magnetic field structure is studied in [19]. The solar sector structure consists of boundaries in the north-south direction, so that on one side of the boundary the large-scale photospheric magnetic field is predominantly directed away from the Sun. On the other side of the boundary, the magnetic field is predominantly directed toward the Sun. For example, the solar wind is designed in such a way that it usually has 2 or 4 sectors (see on http:/ / wso.stanford.edu/SB /SB.htlm (accessed on 4 October 2020)) per solar revolution (27-29 days), within which the magnetic field is usually directed towards or away from the Sun.

In this work, we investigated variations (of the order of the Sun's rotation) in the daily maximum (largest) areas and largest longitudinal widths of the polar and mid-latitude CHs. We supposed that the daily largest area and longitudinal width of the coronal holes are markers characterizing the large-scale structure of the solar magnetic field, which rotates with the Sun. We used the parameters of $\mathrm{CH}$ obtained by two different segmentation methods, originally obtained using the Atmospheric Imaging Assembly instrument onboard the Solar Dynamics Observatory (SDO/AIA) Schou et al. [20], Scherrer et al. [21].

\section{Observations and Data Processing}

To analyze variations in $\mathrm{CH}$ parameters, such as daily largest areas and longitudinal widths, we used data that have been processed by the coronal hole identification method using the multi-thermal emission recognition algorithm (CHIMERA); see in detail [22]. The CHIMERA method was applied to SDO/AIA images obtained in three ultraviolet passbands: 171,193 , and $211 \AA$ to produce the coronal hole recognition. The initial data are presented on: www.solarmonitor.org (accessed on 11 August 2017).

The second method is the region growth algorithm (RGA) described in Wang et al. [23]. This method was improved at the Kislovodsk Mountain Astronomical station (KMAS) [24] for the identification of solar features. We used the same parameters of $\mathrm{CHs}$ obtained from the original data of SDO/AIA observations in the $193 \AA$ A passband. The daily archive of the parameters of each coronal hole produced with the RGA method is available on: www.observethesun.com (accessed on 7 July 2017). The RGA method produced data in the semi-automatic regime, when the operator was controlling the process to remove artefacts related to the location identification of $\mathrm{CHs}$. The same procedure was used to remove artefacts from the data obtained by the CHIMERA method.

The area of coronal holes obtained by the CHIMERA method on the site has units of measurement expressed as a percentage and by the RGA method in millionths of the solar hemisphere. Note that we have recalculated the area units to reflect the curvature of the Sun and now area units are expressed in $\mathrm{mm}^{2}$ for both data sources.

These two methods of the $\mathrm{CH}$ segmentation showed almost the same statistical results for areas in our preliminary studies. The correlation coefficient calculated between the time series for the areas obtained by these two methods is 0.87 . Figure 1 shows one example of the polar coronal holes produced by the two methods mentioned above. 

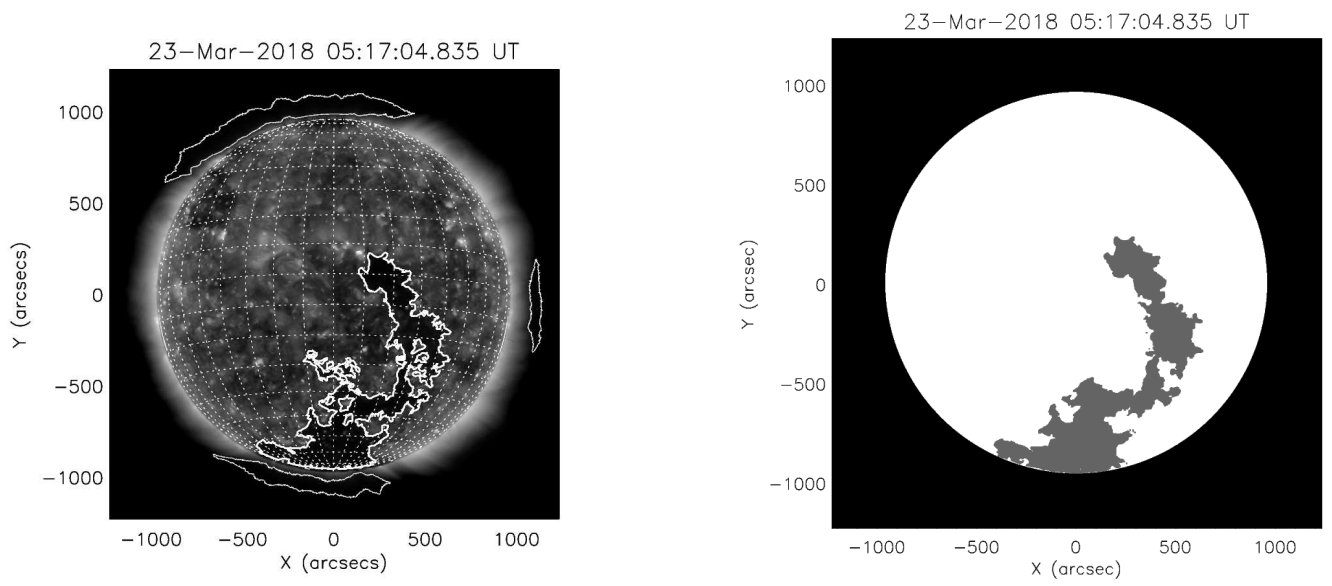

Figure 1. (Left) Coronal hole visible on the solar disk obtained by the CHIMERA method. (Right) The same coronal hole obtained by the RGA method.

We also estimated the lifetime of a typical polar coronal hole and showed the difference in area distribution for the selected observation period (2017-2020). The results are shown in Figures 2 and 3.
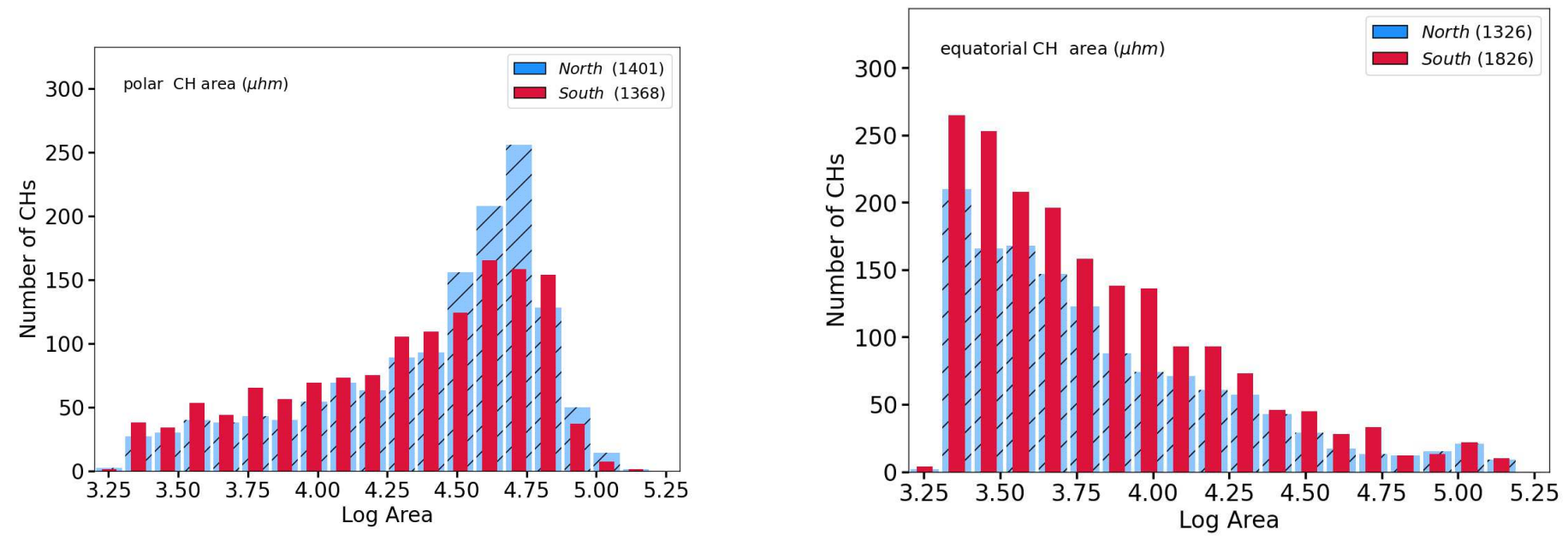

Figure 2. The (left-hand) histogram shows the area distribution of polar coronal holes. The (righthand) histogram shows the area distribution for equatorial coronal holes. The number of coronal holes in each considered zone is indicated in parentheses.

Figure 2 shows two histograms of the distribution of coronal holes areas in different zones of the Sun. The left graph shows the distribution of areas in the polar zones. The right graph shows the area distribution in the mid-latitude zone. Here we see two different types of distribution. In the polar zones, we see an increase in the number of coronal holes with an increase in area, and in the middle (equatorial) zone, a decrease in the number of coronal holes with an increase in area is clearly visible.

We note that the area of the coronal holes presented in Figure 2 is measured in millionth parts of the solar hemisphere $(\mu \mathrm{hm})$ (note that $1 \mu \mathrm{hm}=3.004 \times 10^{6} \mathrm{~km}^{2}$ ).

Now we briefly describe an algorithm for determining the lifetime of coronal holes, results of which we used in Figure 3.

To do this, we found the chains of shifts of coronal holes from day $d$ to $d+1$, measuring their Carrington latitude and longitude every day. We considered the coronal hole the same if the displacement on adjacent days was less than 15 degrees in latitude and less than 20 degrees in longitude. If the meridional distance (or longitude from the central meridian) was less than 70 degrees, then we reassigned the new day's latitude and longitude to coronal holes. Then we looked for them by the new coordinates on the image of the Sun obtained 
for that day. If the coronal hole was not visible the next day, then we continued looking for it in the next $(d+i)$ days, where $d$ now is the last day it was visible, and $i=1,2, \ldots 21$. We explain twenty-one (21) days as follows; for 14 days, we do not see the coronal hole because it is on the invisible side. The position of the coronal hole near the limb is poorly determined, so we add 3.5 days for it to go beyond the western limb. After that coronal hole reappears on the eastern limb at a sufficient distance from it, we again add 3.5 days. Thus, we have 21 days. Using this algorithm, we estimated the lifetime of each coronal hole considered in our study.

Figure 3 shows two histograms of the distribution of the lifetimes of coronal holes. In the case of the polar zone, the lifetime reaches a value of about a thousand days. In the case of middle latitudes, the greatest lifetimes are in the range of 740-1000 days. Thus, using the results presented in Figure 3, we concluded that the observation period used in this study (984 days) is comparable to the longest lifetime of the analyzed coronal holes. Our estimate of the lifetime of CHs agrees with the results in Section 1. It should also be noted that the number of long-lived coronal holes is greater in the southern hemisphere of the Sun than in the northern one. This also indicates the asymmetry in the polar activity in the observable period.
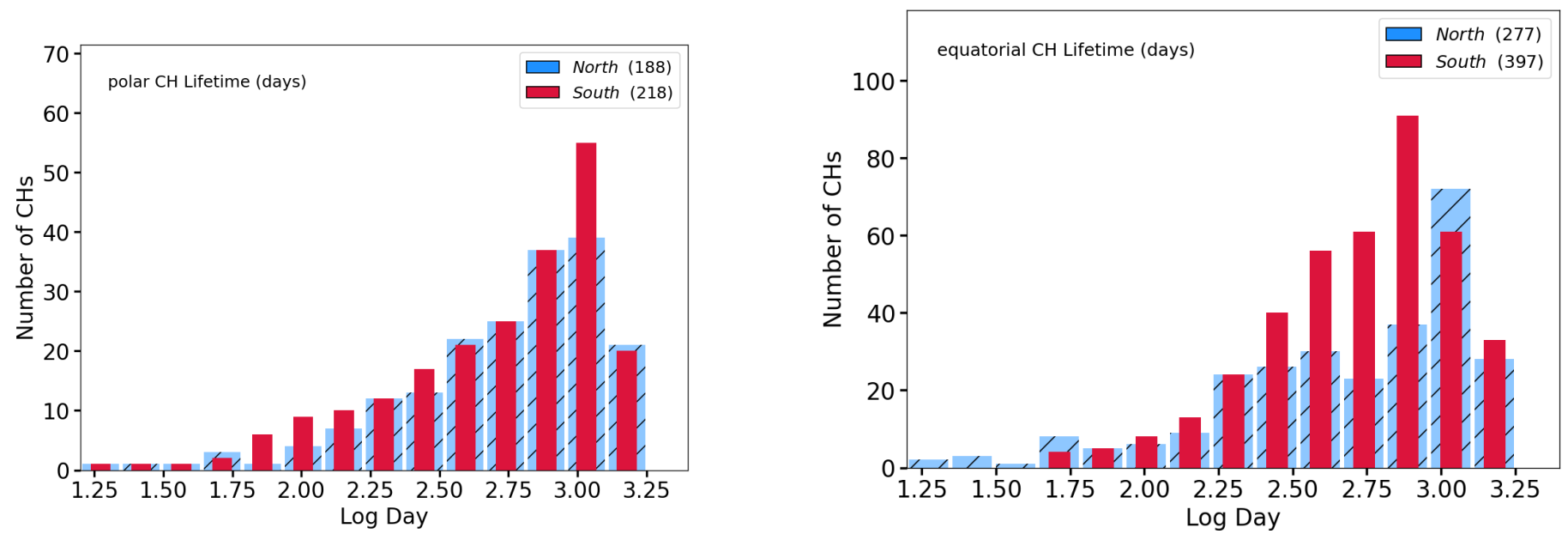

Figure 3. The (left-hand) histogram shows the lifetime distribution for the polar coronal holes. The (right-hand) histogram shows the lifetime distribution for the equatorial coronal holes. The number of coronal holes in each considered zone is indicated in parentheses.

Thus, to study the selected parameters of CHs, a data interval of about 1000 days is sufficient to study the variations in these parameters with periods comparable to the solar revolution.

The starting date of the data series was chosen randomly, on 11 August 2017, with the cadence of one day. We analyzed the series of the daily maximum (or largest) areas and maximum (largest) longitudinal widths of the coronal holes in the polar zones (greater than $50^{\circ}$ of solar latitude in both solar hemispheres) and in the mid-latitude zone (between $\pm 50^{\circ}$ ), separately. In accordance with this definition of the solar zones, the coronal hole presented in Figure 1 is the polar coronal hole, because it has a latitude heliocentric coordinate greater than $50^{\circ}$ of solar latitude. The initial time series consists of the daily maximum parameters of the coronal holes; see Figure 4. The headers above each graph indicate by which method (CHIMERA or RGA) and in which solar zone the corresponding parameters were obtained.

It is important to note that these time series consist of daily maximum (largest) values of the considered parameters. These values do not necessary belong to the same coronal hole. We assume that variations in the daily largest values of these parameters reflect the rotation of the sector structure of the solar magnetic field. 

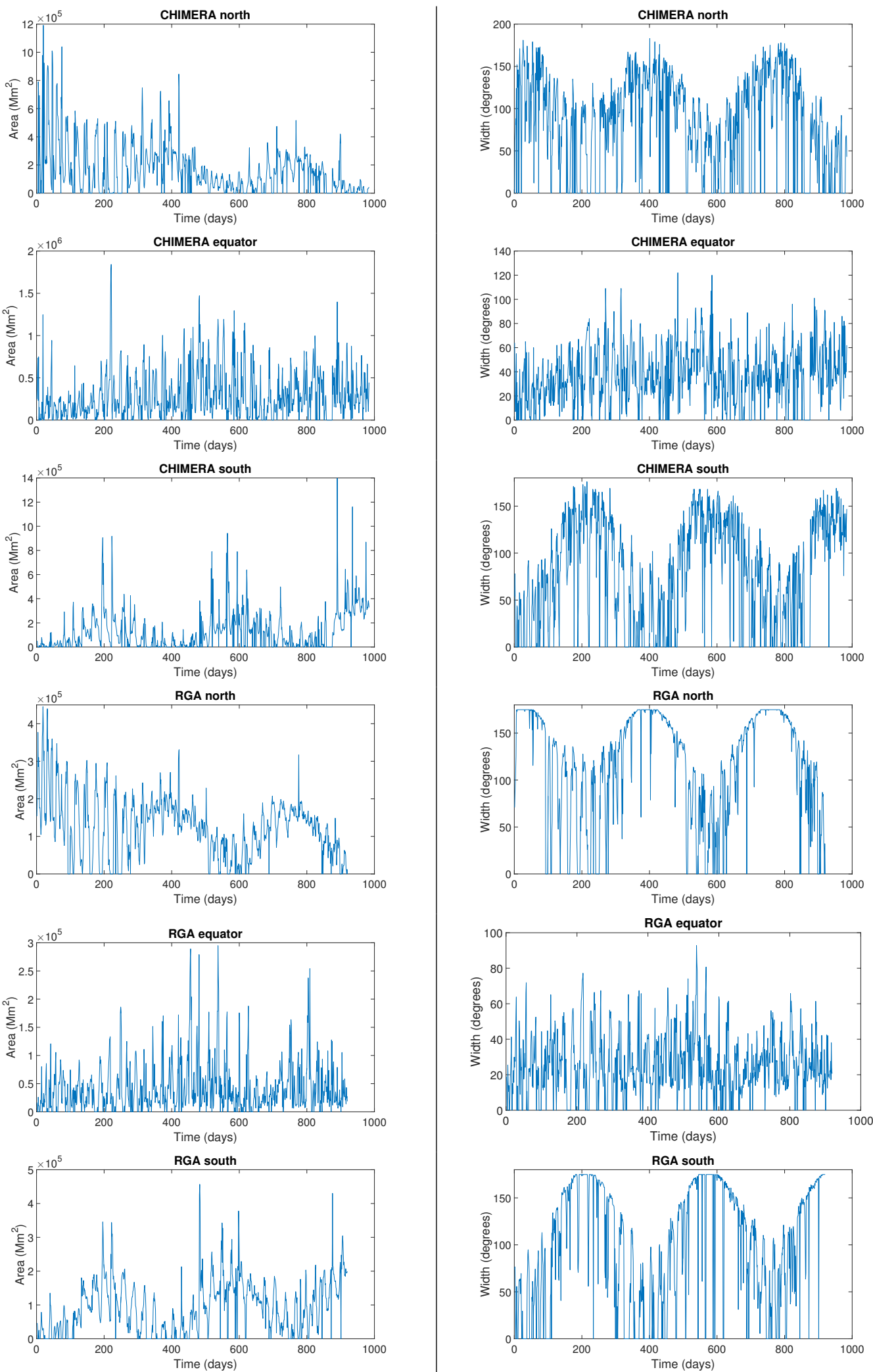

(Area)

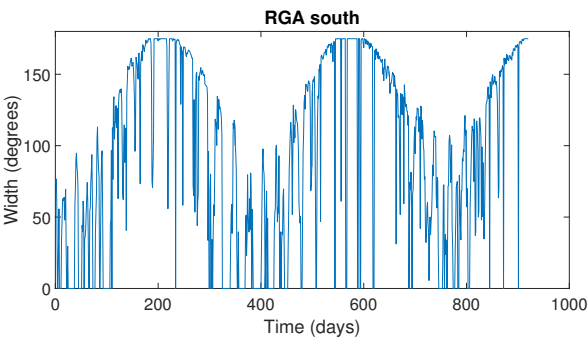

(Width)

Figure 4. Columns represent the initial time series of the daily maximal area and maximal longitudinal width obtained by two methods of the $\mathrm{CH}$ segmentation at different zones of the Sun. 


\section{Results}

Figure 4 shows the original data series. This figure clearly shows a long period of change in the considered parameters of coronal holes. The influence of the trend is especially noticeable in the polar zones. We have considered this lower harmonic with a period of about 350-360 days in the full spectrum as a long-term trend that appears due to a change in the angle $B_{0}$.

Each time series was analyzed using Fourier and wavelet transform. The main results of this study are presented in Figures 5 and 6.

Figure 5 ((Area) column) shows the Fourier spectra obtained for variation in the daily maximum area of coronal holes observed in the northern, equatorial, and southern polar zones of the Sun. Column (Width) shows the Fourier spectra of the daily maximum longitudinal width variations in the analyzed coronal holes. The black bold lines on the graphs show a 95\% confidence level calculated using the $\chi^{2}$ method using the entire spectrum, not just the 40-day part, as shown in Figure 5. Each graph in this figure shows only a 40-day portion of the full spectrum, which is taken in order to show the relatively short periods studied in this work. In the spectra calculated for the largest daily area of coronal holes, we found 14- and 28-day periods of variation in all considered solar zones. In the spectra calculated for the largest daily longitudinal width of coronal holes, we found a 28-day period of variations in all considered solar zones. In addition, for the RGA method of coronal hole segmentation, 14-day periods of variation were found in all zones. Almost all 8-9-day periods of variation of the largest area and width have a small amplitude comparable to the $95 \%$ confidence level or below it.

Before the production of the wavelet analysis, the long-periodic trend (350 days) was subtracted from the original data as follows: each time series was decomposed into 11 internal modes using the empirical mode expansion (EMD) method [25]. One of the reasons for using this method is that we can analyze each mode separately or consider only modes with certain parameters.

Figure 6 shows the wavelet power spectra of the analyzed time series produced with the technique described in Torrence and Compo [26]. Each wavelet diagram consists of three panels. Panels (a), (b), and (c) represent the initial time series, wavelet, and global wavelet spectrum, respectively. The $95 \%$ confidence level is marked with a red dashed line on the global wavelet spectra.

As can be seen from Figure 6, variations in the daily maximum areas of coronal holes and longitudinal width have typical periods of 26-29 and 13-15 days in all three considered zones of the Sun. The period of 8-9 days was determined in the equatorial and southern zones of the Sun as a result of the wavelet transform of the time series obtained by both methods of identifying the coronal hole by both considered parameters. This period has small amplitude comparable to the $95 \%$ confidence level or below it, as in the case of the Fourier results presented in Figure 5.

Panels denoted (b) in Figure 6 show that variations in the maximum area of coronal holes in the north polar zones take place in the observation time interval (1-400) days. Then, these variations become much less pronounced. In the southern and equatorial regions, the variations in the areas of coronal holes are stable throughout the entire period of observations. Such behavior of variations takes place for both methods of segmentation. The behavior of the maximum width of coronal holes is also stable. Thus, the results obtained (see Figure 5 and 6) show that the variations in the considered parameters of coronal holes coincide with the rotation periods 2,4 , and 6 of the sector structure of the large-scale solar magnetic field.

The difference in the maximum number of $\mathrm{CH}$ areas (see Figure 2), and the difference in variations of the largest areas (see Figure 6) in the northern and southern polar zones indicated the asymmetry of polar solar activity.

In addition, we have estimated the lifetime of the recurrent coronal holes in different solar zones; see Figure 3. 

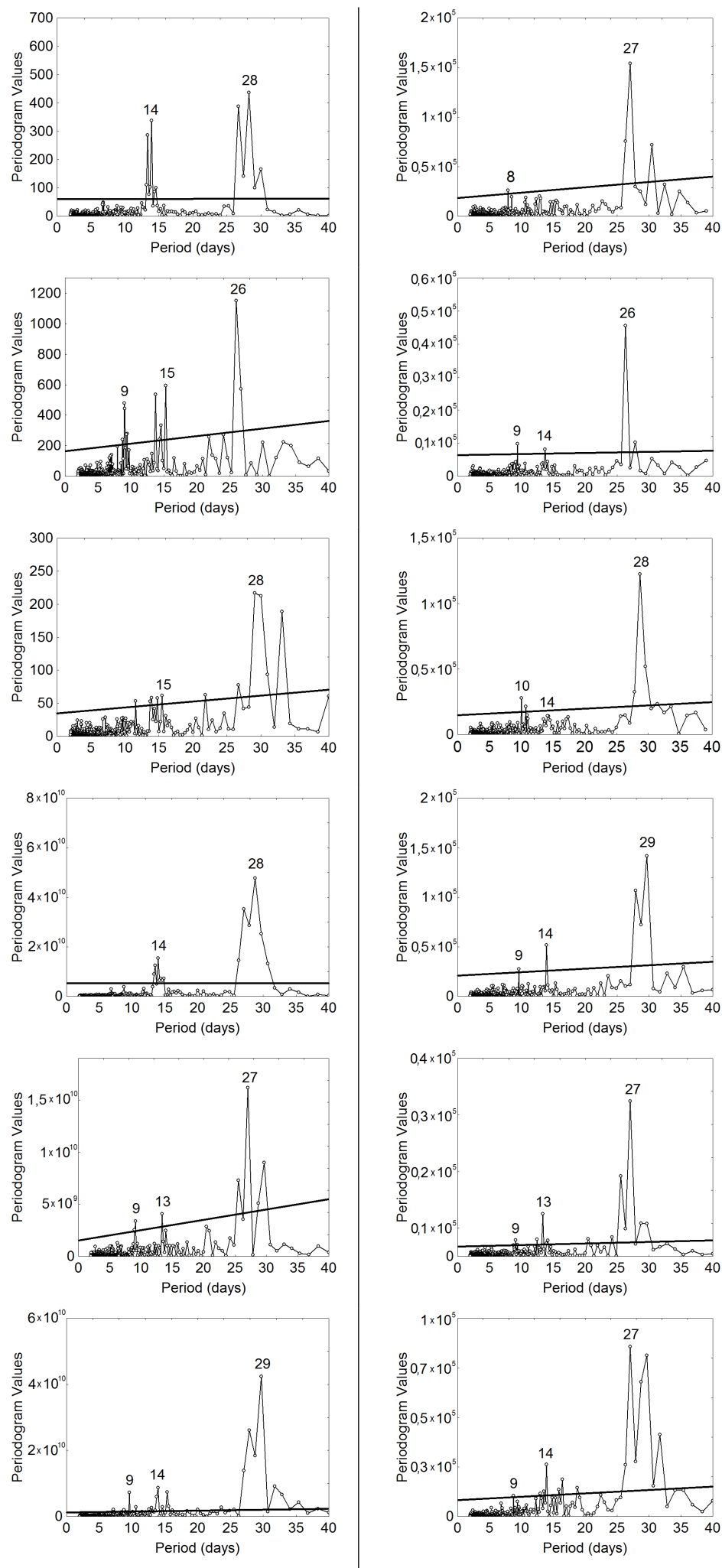

(Area)

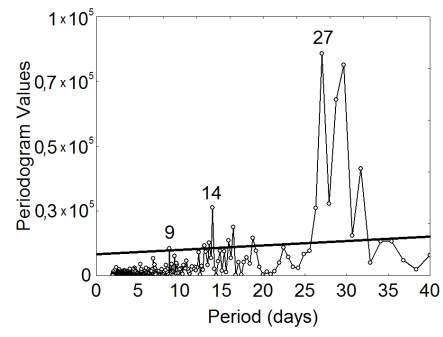

(Width)

Figure 5. Columns (Area) and (Width) represent Fourier spectra for areas and longitudinal width variations in the analyzed $\mathrm{CHs}$. Plots in the figure located in the following order from top to bottom: first-CHIMERA north; second-CHIMERA equator; third-CHIMERA south; fourth-RGA north; fifth-RGA equator; sixth-RGA south. 

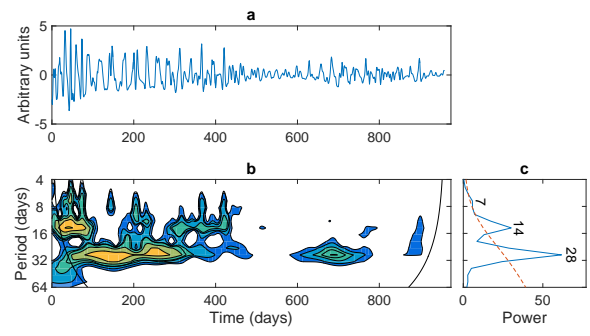

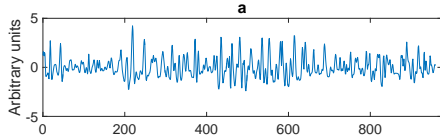
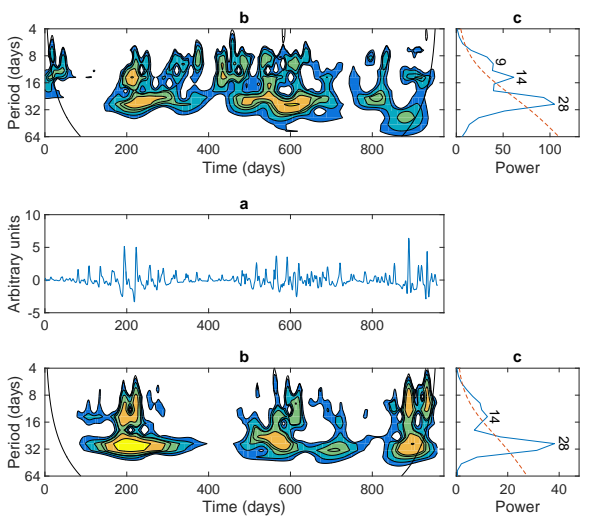

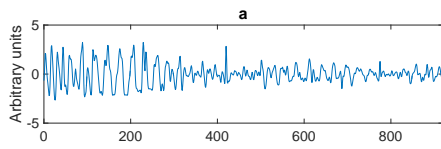

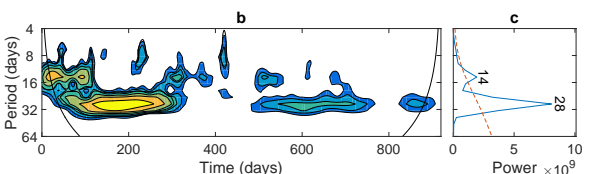

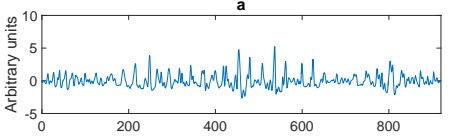
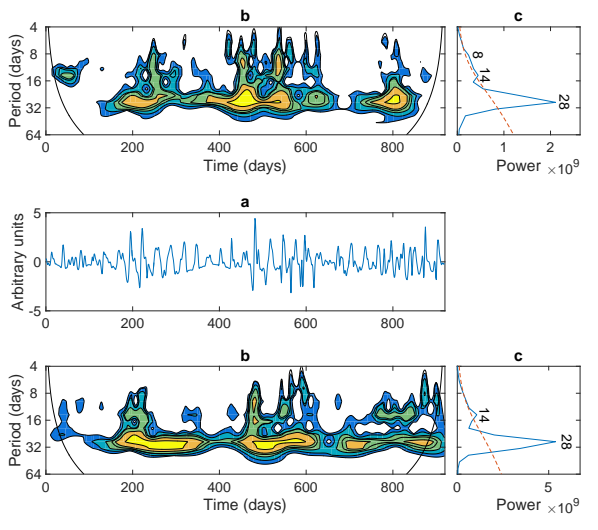

(Area)
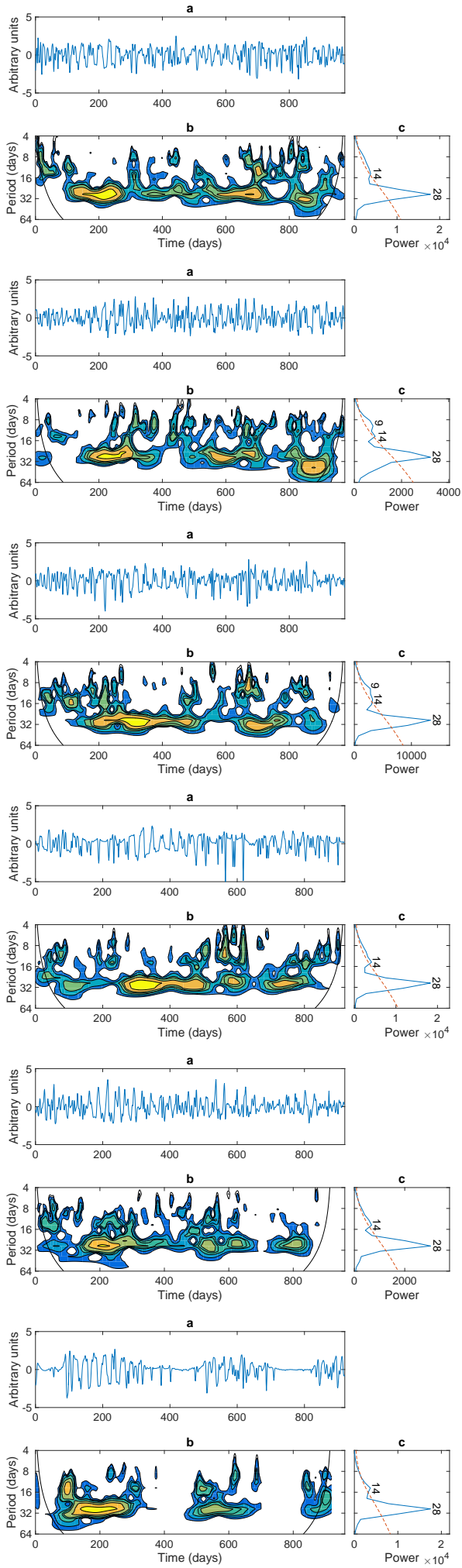

(Width)

Figure 6. Wavelet spectra of daily maximal areas (Area) and longitudinal width (Width) variations in CHs. Plots in the figure located in the following order: first from top: CHIMERA north; second from top: CHIMERA equator; third from top: CHIMERA south; fourth from top: RGA north; fifth from top RGA equator; sixth from top RGA south. 


\section{Discussion and Conclusions}

Now we will try to elucidate the origin of the quasi-periodic variations in the considered parameters of the $\mathrm{CHs}$. The typical periods of $\mathrm{CH}$ variations are $26-29,13-15$, and 8-9 days. It is important to emphasize that the spectral characteristics (periodicity) of time series obtained by two different methods of the identification of $\mathrm{CH}$ boundaries do not differ.

The period of 26-28 days is the period of the solar rotation obtained by many authors; for example, see [27]. These results were obtained for the equatorial zone of the Sun. In our work, this period was defined in all considered solar zones. It could be suggested that coronal holes, at least the largest one, rotate more rigidly than the photosphere rotation rate obtained by the Doppler shift method and the method of helioseismology; see details in Bagashvili et al. [9]. In the same article, the authors proclaimed that the rotation rate of the corona was connected to the rotation rate of the solar interior.

There are some other possible explanations for the obtained periods. For example, cyclic variation studies of the large-scale solar magnetic field, provided by [28,29], have shown the rotation periods of $13.64,26.86$, and 9 days. These periods have been explained by the four-, two-, and six-sector structures, respectively. All these periods were obtained for the mid-latitude zone of the Sun.

Thus, if we consider $\mathrm{CHs}$ as a marker of the large-scale magnetic field structure, we can suggest that periods of 26-28, 13-14, and 8-9 days are the typical rotation periods for the two- , four-, and six sector structures. It should be noted that the 8-9 day period has a small amplitude in the polar zones of the Sun. It probably relates to the great difficulties of observations associated with the projection effects in the polar zones.

The article published by Fossat et al. [30] reported the discovery of g-mode splitting and the fast rotation of the solar core with a period of about 7 days. According to the authors, the mean weighted rotation of their kernels has a period of about 9 days. Considering this result, we can suggest that the period of 8-9 days found in our work could also be associated with the rotation of the splitting kernels of the lowest gravitational modes. This can happen in the following scenario: the rotation of the core affects the intensity of the photosphere, which is revealed by the variations in total solar irradiance (TSI) [31]. Coronal holes are based on the photosphere, which is their lower boundary. In turn, the photosphere senses fluctuations defined in the solar core with a period of 9 days. The magnetic field from the radiant zone modulated by the core breaks through the convective zone. This effect is reflected to the convective transfer, and therefore the integral brightness of the photosphere has a period of 9 days.

In our further work, we would like to continue the study of variations in the considered parameters of coronal holes on a time interval of the order of the solar cycle.

Author Contributions: Conceptualization, A.R. and A.S.; methodology, V.S., F.A.-H., A.R., and A.T.; software, I.Z.; formal analysis, V.S. and P.S.; investigation, A.R.; writing-original draft preparation, A.R. and V.S. All authors have read and agreed to the published version of the manuscript.

Funding: This research was funded by Solov'ev A and was supported by the Ministry of Science and Higher Education of the Russian Federation under the grant 075-15-2020-780 (N13.1902.21.0039). Tlatov A. was supported by the grant RFBR 18-52-34004. Smirnova V. was supported by the Ministry of Science and Higher Education of the Russian Federation (project No. 0831-2019-0006) and by the Russian Foundation for Basic Research grant No. 20-52-26006.

Data Availability Statement: Not applicable.

Acknowledgments: Authors thank the SDO team for the possibility of using materials from the Solar Dynamic Observatory.

Conflicts of Interest: The authors declare no conflict of interest. 


\section{References}

1. Cranmer, S.R. Coronal Holes. Living Rev. Sol. Phys. 2009, 6, 3. [CrossRef] [PubMed]

2. Bale, S.D.; Badman, S.T.; Bonnell, J.W.; Bowen, T.A.; Burgess, D.; Case, A.W.; Cattell, C.A.; Chandran, B.D.G.; Chaston, C.C.; Chen, C.H.K.; et al. Highly structured slow solar wind emerging from an equatorial coronal hole. Nature $2019,576,237-242$. [CrossRef] [PubMed]

3. Krieger, A.S.; Timothy, A.F.; Roelof, E.C. A Coronal Hole and Its Identification as the Source of a High Velocity Solar Wind Stream. Sol. Phys. 1973, 29, 505-525. [CrossRef]

4. Heinemann, S.G.; Jerčić, V.; Temmer, M.; Hofmeister, S.J.; Dumbović, M.; Vennerstrom, S.; Verbanac, G.; Veronig, A.M. A statistical study of the long-term evolution of coronal hole properties as observed by SDO. Astron. Astrophys. 2020, 638, A68. [CrossRef]

5. Heinemann, S.G.; Temmer, M.; Heinemann, N.; Dissauer, K.; Samara, E.; Jercic, V.; Hofmeister, S.J.; Veronig, A.M. VizieR Online Data Catalog: Coronal hole parameters (Heinemann+, 2019). VizieR Online Data Cat. (Other) 2019, 0580, J/other/SoPh/294.

6. Heinemann, S.; Pomoell, J.; Temmer, M.; Bourdin, P. Life-time evolution and magnetic structure of coronal holes. In Proceedings of the 43rd COSPAR Scientific Assembly, Sydney, Australia, 28 January-4 February 2021; Volume 43, p. 1024.

7. Hofmeister, S.J.; Veronig, A.; Reiss, M.A.; Temmer, M.; Vennerstrom, S.; Vršnak, B.; Heber, B. Characteristics of Low-latitude Coronal Holes near the Maximum of Solar Cycle 24. Astrophys. J. 2017, 835, 268. [CrossRef]

8. Hofmeister, S.J.; Utz, D.; Heinemann, S.G.; Veronig, A.; Temmer, M. Photospheric magnetic structure of coronal holes. Astron. Astrophys. 2019, 629, A22. [CrossRef]

9. Bagashvili, S.R.; Shergelashvili, B.M.; Japaridze, D.R.; Chargeishvili, B.B.; Kosovichev, A.G.; Kukhianidze, V.; Ramishvili, G.; Zaqarashvili, T.V.; Poedts, S.; Khodachenko, M.L.; et al. Statistical properties of coronal hole rotation rates: Are they linked to the solar interior? Astron. Astrophys. 2017, 603, A134. [CrossRef]

10. Cadavid, A.C.; Miralles, M.P.; Romich, K. Comparison of the Scaling Properties of EUV Intensity Fluctuations in Coronal Hole and Quiet-Sun Regions. Astrophys. J. 2019, 886, 143. [CrossRef]

11. Cranmer, S.R.; Winebarger, A.R. The Properties of the Solar Corona and Its Connection to the Solar Wind. Annu. Rev. Astron. Astrophys. 2019, 57, 157-187. [CrossRef]

12. Cranmer, S.R. Alfven Waves in the Solar Corona and Solar Wind: An Updated Energy Budget. In Proceedings of the American Astronomical Society Meeting Abstracts \#235, Honolulu, HI, USA, 4-8 January 2020; Volume 235, p. 149.08.

13. Nakagawa, Y.; Nozawa, S.; Shinbori, A. Relationship between the low-latitude coronal hole area, solar wind velocity, and geomagnetic activity during solar cycles 23 and 24. Earth Planets Space 2019, 71, 24. [CrossRef]

14. Hara, H. Nonthermal Motions in a Polar Coronal Hole Measured with Hinode/EIS during an on-Orbit Partial Solar Eclipse on 2017 August 21. Astrophys. J. 2019, 887, 122. [CrossRef]

15. Mazumder, R.; Bhowmik, P.; Nandy, D. Properties of Coronal Holes in Solar Cycle 21-23 using McIntosh archive. IAU Symp. 2018, 340, 187-188. [CrossRef]

16. Bravo, S.; Otaola, J.A. Polar Coronal Holes and the Sunspot Cycle-A New Method to Predict Sunspot Numbers. Sol. Phys. 1989, 122, 335-343. [CrossRef]

17. Hofer, M.Y.; Storini, M. Repeated Structures Found After the Solar Maximum in the Butterfly Diagrams of Coronal Holes. In American Institute of Physics Conference Series; Ten, S.W., Velli, M., Bruno, R., Malara, F., Bucci, B., Eds.; American Institute of Physics: Melville, NY, USA, 2003; Volume 679, pp. 234-237. [CrossRef]

18. Ikhsanov, R.N.; Tavastsherna, K.S. Latitude-temporal evolution of coronal holes in cycles 21-23. Geomagn. Aeron. 2015, 55, 877-883. [CrossRef]

19. Stepanian, N.N.; Shtertser, N.I. Polar coronal holes in the solar activity cycle. Adv. Space Res. 2015, 55, 795-797. [CrossRef]

20. Schou, J.; Scherrer, P.H.; Bush, R.I.; Wachter, R.; Couvidat, S.; Rabello-Soares, M.C.; Bogart, R.S.; Hoeksema, J.T.; Liu, Y.; Duvall, T.L.; et al. Design and Ground Calibration of the Helioseismic and Magnetic Imager (HMI) Instrument on the Solar Dynamics Observatory (SDO). Sol. Phys. 2012, 275, 229-259. [CrossRef]

21. Scherrer, P.H.; Schou, J.; Bush, R.I.; Kosovichev, A.G.; Bogart, R.S.; Hoeksema, J.T.; Liu, Y.; Duvall, T.L.; Zhao, J.; Title, A.M.; et al. The Helioseismic and Magnetic Imager (HMI) Investigation for the Solar Dynamics Observatory (SDO). Sol. Phys. 2012, 275, 207-227. [CrossRef]

22. Garton, T.M.; Gallagher, P.T.; Murray, S.A. Automated coronal hole identification via multi-thermal intensity segmentation. J. Space Weather Space Clim. 2018, 8, A02. [CrossRef]

23. Wang, Q.; Song, X.; Jiang, Z. An Improved Image Segmentation Method Using Three-dimensional Region Growing Algorithm. In Proceedings of the 2013 International Conference on Information Science and Computer Applications, Changsha, China, 8-9 November 2013; Atlantis Press: Amsterdam, The Netherlands, 2013; pp. 148-152. [CrossRef]

24. Tlatov, A.G.; Vasil'eva, V.V.; Makarova, V.V.; Otkidychev, P.A. Applying an Automatic Image-Processing Method to Synoptic Observations. Sol. Phys. 2014, 289, 1403-1412. [CrossRef]

25. Huang, N.E.; Shen, Z.; Long, S.R.; Wu, M.C.; Shih, H.H.; Zheng, Q.; Yen, N.C.; Tung, C.C.; Liu, H.H. The empirical mode decomposition and the Hilbert spectrum for nonlinear and non-stationary time series analysis. Proc. R. Soc. Lond. Ser. A 1998, 454, 903-998. [CrossRef]

26. Torrence, C.; Compo, G.P. A practical guide to wavelet analysis. Am. Meteorol. Soc. 1998, 79, 61-78. [CrossRef]

27. Hiremath, K.M.; Hegde, M. Rotation Rates of Coronal Holes and their Probable Anchoring Depths. Astrophys. J. 2013, 763, 137. [CrossRef] 
28. Obridko, V.N.; Shelting, B.D. Structure of the Heliospheric Current Sheet derived for the interval 1915-1916. Sol. Phys. 1999, 184, 187-200. [CrossRef]

29. Vasil'Eva, V.V.; Makarov, V.I.; Tlatov, A.G. Rotation Cycles of the Sector Structure of the Solar Magnetic Field and Its Activity. Astron. Lett. 2002, 28, 199-205. [CrossRef]

30. Fossat, E.; Boumier, P.; Corbard, T.; Provost, J.; Salabert, D.; Schmider, F.X.; Gabriel, A.H.; Grec, G.; Renaud, C.; Robillot, J.M.; et al. Asymptotic g modes: Evidence for a rapid rotation of the solar core. Astron. Astrophys. 2017, 604, A40. [CrossRef]

31. Efremov, V.I.; Parfinenko, L.D.; Solov'ev, A.A. Global long-term oscillations of the Sun observed by SORCE, SOHO and SDO. Astrophys. Space Sci. 2018, 363, 257. [CrossRef] 